\title{
Analysis of the Role and Process of Emotion Regulation in Relation with Generalized Anxiety Disorder
}

\author{
Ankita Vayalapalli ${ }^{1, *}$, Amoolya Vayalapalli ${ }^{2}$ \\ ${ }^{1}$ Northview High School, Georgia, USA \\ ${ }^{2}$ Augusta University, Georgia, USA
}

Copyright $(2018$ by authors, all rights reserved. Authors agree that this article remains permanently open access under the terms of the Creative Commons Attribution License 4.0 International License

\begin{abstract}
This paper analyzes the role of emotion regulation in patients diagnosed with Generalized Anxiety Disorder (GAD). Although there are various types of emotion regulation, this paper studies cognitive reappraisal and expressive suppression specifically because of their prevalence and imperative role in the behavioral aftermath of emotion. Cognitive reappraisal is studied as an antecedent-focused strategy to control and modify the meaning of a situation, whereas expressive suppression is analyzed as a response-focused strategy that alters behaviors associated with situations that elicit emotion. Patients diagnosed with GAD use emotion regulation as both a defensive strategy and a coping mechanism. Through this understanding, the role of Emotion Regulation Therapy (ERT) in conjunction with Cognitive Behavioral Therapy (CBT) is depicted. Emotion Regulation Therapy is also proposed as an alternative to CBT because of its ability to cater specifically to individuals with anxiety disorders. This paper studies the implications of the usage of emotion regulation both as a coping mechanism that is self-employed and therapeutically used by health professionals.
\end{abstract}

Keywords Generalized Anxiety Disorder, Emotion Regulation, Emotion Regulation Therapy, Cognitive Behavioral Therapy, Cognitive Reappraisal, Emotion Suppression, Anxiety Disorders, Amygdala

\section{Generalized Anxiety Disorder}

Generalized Anxiety Disorder (GAD) is a psychological disorder that affects roughly $3.1 \%$ of the population in the United States [2]. It is considered treatable by the use of antidepressants and behavioral therapy. Although, according to the Anxiety and Depression Association of America (ADAA), only $43.2 \%$ of people with the disorder are actually receiving treatment. Patients with GAD tend to worry "excessive(ly) and inappropriate(ly) about issues such as health, money, work, or family [1]. They also present with physical and psychological symptoms such as tachycardia and tremor and psychological restlessness, fatigue, and irritability [14]. Because of its systemic manifestation, GAD makes it somewhat difficult to live a high quality of life without being encumbered with the psychological stress that can manifest physiologically. GAD often coexists with other psychiatric disorders and is comorbid with major depressive disorder (MDD), obsessive-compulsive disorder (OCD), and panic disorder. This is usually the case with a wide array of psychiatric disorders because of a high degree of genetic and environmental correlations between the pathogenesis of many of them [1]. Women tend to be diagnosed twice as much as men, but research shows that this disparity could be a result of social pressure that causes men to under-report or not seek treatment. The exact cause of GAD is unknown, but patients from a lower socioeconomic status and those with stressful childhoods tend to have a higher correlation with a diagnosis of the disorder [18].

\section{Stress, Anxiety and Fear}

High levels of stress, anxiety, and fear are positively correlated with a diagnosis of GAD. Stress can be defined as either a "stimulus that happens to a person" or a "response characterized by physiological arousal and negative effect". It is often accompanied by biochemical, physiological, and behavioral changes as well [7]. Stress is both a motivator for actions and a cause for anxiety because of its two-fold nature. The main hormones that control stress are cortisol, epinephrine, norepinephrine, corticotropin-releasing hormone, and adrenocorticotropic hormone. In a study conducted by the University of Michigan's Department of Psychiatry, stress was shown to be a latent trait that occurred only when the situation "presented itself". The release of adrenocorticotropic hormone (ACTH) via the hypothalamus assists with acute actions during various "threats and opportunities" [5]. 
Constant stress caused by any of a myriad of factors can lead either to the pathogenesis of GAD or presents as a symptom as a result.

Anxiety and fear are often confused for each other: the former arises from a perceived threat from the future, while the latter is caused by a real threat at the present time. Anxiety is characterized by a "prolonged state of tension, worry, and apprehension regarding uncertain, and potentially negative, future events" [5]. Fear is defined as an immediate response to a certain situation that induces "tension, worry, or apprehension" [5]. Both fear and anxiety are "an organism's defensive response" [12] towards potential "sources of danger" and serve an "evolutionary purpose" [21] because they help increase survival chances in adverse conditions. The issue with excessive anxiety and fear is that the modern population of human beings does not normally encounter (on a daily basis) the extraneous situations in which life-or-death decisions need to be made to protect oneself physically from harm. Anthropological history details extensive circumstances in which survival rates for humans were much lower and man had to hunt for food and protection, risking death [15]. Since anxiety is known to be genetic, it can be presumed that natural selection played a role in the passing down of it, because such traits were favorable and would often lead to reproductive success [11]. Adults with a greater sense of anxiety and fear would be able to use their instincts to escape imminent danger. They would then survive and live to bear children, passing on their traits. Although the majority of the population does not currently experience these situations, the neurobiological responses are intact and these latent tendencies are "highly exaggerated" in certain individuals [8].

\section{Emotion Regulation}

Defining emotion is difficult since it is such an abstract concept. It usually satisfies three criteria: duration, cognitive and somatic changes, and some sort of appraisal or assessing in the aftermath [25]. Although emotion is instinctive, it can be regulated and manifest or present in a myriad of ways-- which can then be studied to understand coping mechanisms people use to deal with emotion-eliciting situations.

Emotion regulation ranges from, but is not restricted to, cognitive reappraisal, distraction, avoidance, escape, suppression, emotion and problem-focused coping, and the use of substances to enhance or blunt emotional experience. In this paper, we analyze cognitive appraisal and expressive suppression specifically because of their prevalence in association to GAD [16].

Cognitive reappraisal allows individuals to "implement and produce interpersonal behavior" that is properly centered on social communications and is generally perceived as "emotionally engaging and responsive" [4]. Cognitive reappraisal manifests through reinterpretation of a certain positive or negative emotional stimulus. For example, if a runner does not finish a marathon that he or she attempted to complete, they could be sad or frustrated at first. If they employed cognitive reappraisal, they would reevaluate the situation and analyze what could have been done differently and how he or she can change in the future to prepare for a more favorable outcome. Cognitive reappraisal is a two-step process that involves 1) the recognition of the negative emotion-eliciting circumstance and the consequent negative emotion and 2) the attempt to reinterpret or reevaluate the circumstance and emotion associated with it through a more positive light [28]. Both "stress and depression" can be alleviated through the consistent use of "positive reappraisal" [17]. The implications behind this are important because, as aforementioned, patients with GAD experience chronic low to high grade stress. Furthermore, GAD is also comorbid with major depressive disorder and other psychiatric disorders as well. Cognitive reappraisal is described as a "latent strategy", yet in the past 20-30 years, there has been research that substantiates meditation as a method to attain cognitive control at will [3]. Although meditation is harder to implement therapeutically, simply being aware of the situation and understanding that the feeling of anxiety will be temporary has proven to be useful to decrease the amount of stress in patients [22]. It modifies the behavioral or physiological response to an emotional stimulus.

Expressive suppression is characterized by the modification of "behavioral or physiological response(s)" to a specific emotional stimulus. It is usually characterized by the escape or avoidance of negative emotions [20]. This emotion regulation strategy employs "response modification" as opposed to "cognitive modification" that presents in cognitive reappraisal. For example, if a person is watching a gory scene with his friends, instead of flinching or grimacing, he forces his expression to seem 'normal'. Expressive suppression is often cited as a manipulation of facial expressions and body language in order to avoid a certain emotion. Many studies also refer to it as a societal caused coping mechanism because of the subconscious behaviors that can "impede... interpersonal functioning" [20]. By acting as if something did not occur, people who suppress their expressions believe that they can avoid the consequences of a situation that is emotionally painful. Of the two methods studied in this paper, suppression is the less effective long-term regulatory style because frequent usage can be detrimental to both psychological and physical health [27].

In a study conducted by Fucito et al., chronic smokers were separated and grouped into cognitive reappraisers and expressive suppressors with a control group that was not explicitly instructed to use emotion regulation [10]. The study's intent was to measure the frequency of smoking in smokers who attempted to quit by using either cognitive reappraisal or expressive suppression. The group that was instructed to use cognitive reappraisal showed greater rates 
of improvement (measured by lower carbon monoxide levels), a greater positive mood, and decreased symptoms of depression. This data, although related to more physiological symptoms and changes can be extrapolated and studied in relation to neurological disorders and neurobiological symptoms.

\section{Emotion Regulation in Generalized Anxiety Disorder}

Because GAD is characterized by the onset of intense anxiety for extended periods, a natural and instinctive response is to attempt to regulate the negative emotions that ensue. Emotion regulation in response to GAD can be studied through a neurobiological perspective; the neuroscience behind the mechanisms can be identified and therefore analyzed.

\section{Neurobiological Basis}

The brain regions associated with emotion regulation are the medial prefrontal cortex (mPFC), anterior cingulate cortex (ACC), ventral striatum (VS), dorsolateral prefrontal cortex (dIPFC), inferior frontal gyrus triangularis (iFGTR), ventrolateral prefrontal cortex (vIPC), orbitofrontal cortex (OFC), anterior insula and the amygdala [6]. The brain regions associated with GAD are the amygdala, hippocampus, and the hypothalamus. The amygdala, specifically the amygdaloid nuclei, processes "fear conditioning and expresses fear" responses. The hippocampus then uses contextual processing and the hypothalamus releases hormones to the rest of the body, creating a systemic response. The neurotransmitters targeted in anxiety disorders are gamma-aminobutyric acid (GABA), serotonin, dopamine, and epinephrine [23]. Anxiety is carried out by the limbic system, which consists of mainly the amygdala, hippocampus, thalamus, hypothalamus, basal ganglia, and the cingulate gyrus [26].

The dIPFC, IFGTr, vIPC, mPFC, ACC, and OFC are all cortical regions of the brain, meaning they are part of the cerebral complex composed of the frontal, parietal, temporal, and occipital lobes. The cortical regions control higher brain functions such as thought and action [24]. The amygdala, hippocampus, ventral striatum, and basal ganglia are part of the subcortical regions of the brain. The subcortex, composed of the forebrain, midbrain, and hindbrain, enables "instinctual emotional processing" and "dictates...the affective forces that ruled behavior prior to cortical evolution" [19].

The shared region of the brain in the mechanism and exhibition of anxiety is the amygdala. The amygdala "processes emotionally salient" external stimuli and initiates a specific behavioral response. It expresses fear, aggression, and other species-specific defensive behaviors. The amygdala coordinates fear, memory, and emotion, while the hypothalamus elicits hormones to the bloodstreams that trigger the appropriate physical responses-- increased blood pressure, heart rate, et cetera [26][29].

\section{Emotional Regulation Therapy}

The neurobiological basis of emotion regulation and GAD connects the two, making their relationship one that can be studied. The amygdala, a part of the subcortical region of the brain, is involved in emotional processing and is integral to understanding regulation in GAD patients as well. Cognitive reappraisal and expressive suppression are the foils of each other, which explains the vastly different outcomes that occur when one or the other is employed.

Cognitive Behavioral Therapy (CBT) is the current treatment that is generally used to treat an array emotional disorder. It is a combination of "short-term" therapy and "skills-focused treatment(s)" to alter maladaptive emotional responses. It mainly teaches coping strategies in order to change thinking patterns and accept the situation that causes the negative response. Although it is widely used, it possesses some shortcomings, especially in treating anxiety disorders. In a study conducted by the Boston University Department of Psychology by Dr. Stefan G. Hoffman and Dr. Jasper A. Smits, 1,165 patients with anxiety disorders that fit DSM-3R or DSM-IV were evaluated [13]. There was a control group of patients that did not undergo CBT and those that did, and through their research, Hoffman and Smits determined that although "cognitive-behavioral therapy has been systematically used for...anxiety disorders", it "needs considerable improvement" [13]. In another study conducted by the University of California, Los Angeles (UCLA), 40 children with an anxiety disorder were provided CBT and the results were analyzed to determine its effectiveness. The researchers concluded that although it was effective to an extent, CBT "needs to be expanded to address...more clinical characteristics that may cause or compound anxiety symptoms" [27]. In light of such responses, other forms of therapy should be explored in order to tailor treatment specifically for Generalized Anxiety Disorder. One such form of therapy is Emotion Regulation Therapy (ERT). ERT is an efficacious form of therapy for patients with GAD specifically. Its success stems from its multifaceted approach: ERT combines the traditional aspects of CBT with "mechanism-targeted intervention" while focusing on "patterns of processing and behavioral" dysfunction [9].

ERT targets motivational, regulatory, and contextual learning mechanisms because these processes become dysfunctional in patients with GAD. Rather than a normative functional response, patients with GAD experience "heightened [emotional] intensity" and utilize "maladaptive emotional regulation" that favors protection over promotion. Instead of progressing and overcoming 
feelings of anxiety and fear, people with GAD employ more or less ineffective defense mechanisms, which cause a constant circle of low-grade anxiety that cannot be overcome unless it is explicitly treated rather than implicitly avoided temporarily. ERT treats by focusing on the core thematic issues patients with GAD experience and also has a relapse prevention aspect that ensures that progress is sustainable and long-term, rather than short-lived asymptomatic periods that CBT can induce [24]. Although the results are promising for instituting ERT as a commonly used practice, more concrete guidelines need to be placed concerning the scope of the treatment.

\section{Discussion}

In an era of stigma and costly healthcare, it is imperative that mental disorders are thoroughly researched and treatment strategies are both affordable and effective. Generalized Anxiety Disorder is common enough that it requires a substantial amount of research that needs to be done in order to confirm existing schools of thought or challenge current treatments to better understand the complexity of the disorder and properly provide care for those who have it. In this paper, the role of emotion regulation in patients with GAD was analyzed through several aspects. Cognitive reappraisal and expressive suppression were first studied as a self-employed coping mechanism and the former proved to be a more long-term solution than the latter because it alters perception rather than ignoring reality.

To fully understand the cause and nature of GAD, its anthropological history should be examined as well. Although it is often overlooked, anxiety was first experienced as an adaptive-response to external factors that were life-and-death, such as starvation and the threat of being attacked. Anxiety and stress, which are hereditary, were naturally selected for because they increased the chance of survival. In the 21st century, however, such high levels of anxiety and stress are maladaptive because they can detract from social interactions and a person's overall health.

Furthermore, Cognitive Behavioral Therapy was analyzed: although it may be effective in treating certain disorders, it has many shortcomings when used to treat anxiety disorders like GAD. CBT, as initially practiced, is limited in its scope and should be altered or used in conjunction with other, more specialized forms of therapy. Emotion Regulation Therapy (ERT) was discussed as a probable alternative because of its comprehensive treatment and its ability to cater to the subset of anxiety related disorders. It borrows the "mechanism-targeted intervention" element from CBT, but then expands to include a strong focus on relapse prevention and experiential exposure. Although it may not address all the concerns that envelop GAD, it is a significant improvement from the commonly used traditional behavioral therapy.
The implications behind this could potentially change the way patients with GAD are treated. Although change has been historically met with resistance, psychiatrists, psychologists, and therapists can simply implement portions of ERT to make their sessions more effective.

\section{Acknowledgements}

Special thanks to Dr. Rangaprakash Deshpande, fellow at the Department of Psychiatry and Behavioral Sciences at UCLA, for his mentorship and editing, which greatly improved the quality of this manuscript.

\section{REFERENCES}

[1] Anttila, V., Bulik-Sullivan, B., Finucane, H. K., Walters, R. K., Bras, J., Duncan, L., Neale, B. M. (2018). Analysis of shared heritability in common disorders of the brain. Science, 360(6395). doi:10.1126/science.aap 8757

[2] Bandelow, B., \& Michaelis, S. (2015). Epidemiology of anxiety disorders in the 21 st century. Dialogues in Clinical Neuroscience, 17(3), 327-335.

[3] Chan, R. W., Immink, M. A., \& Lushington, K. (2017). The influence of focused-attention meditation states on the cognitive control of sequence learning. Consciousness and Cognition, 55, 11-25. doi:10.1016/j.concog.2017.07.004

[4] Cutuli, D. (2014). Cognitive reappraisal and expressive suppression strategies role in the emotion regulation: an overview on their modulatory effects and neural correlates. Frontiers in Systems Neuroscience, 8, 175. http://doi.org/10.3389/fnsys.2014.00175

[5] Duval, E. R., Javanbakht, A., \& Liberzon, I. (2015). Neural circuits in anxiety and stress disorders: a focused review. Therapeutics and Clinical Risk Management, 11, 115-126. http://doi.org/10.2147/TCRM.S48528

[6] Etkin, A., Egner, T., \& Kalisch, R. (2011). Emotional processing in anterior cingulate and medial prefrontal cortex. Trends in Cognitive Sciences, 15(2), 85-93. http://doi.org/10.1016/j.tics.2010.11.004

[7] Folkman, S. (2013). Stress: Appraisal and Coping. Encyclopedia of Behavioral Medicine, doi:10.1007/978-1-4419-1005-9 215

[8] Friedman, A. L., Burgess, A., Ramaseshan, K., Easter, P., Khatib, D., Chowdury, A., Diwadkar, V. A. (2017). Brain network dysfunction in youth with obsessive-compulsive disorder induced by simple uni-manual behavior: The role of the dorsal anterior cingulate cortex. Psychiatry Research, 260, 6-15.

http://doi.org/10.1016/j.pscychresns.2016.12.005

[9] Fresco, D. M., Mennin, D. S., Heimberg, R. G., \& Ritter, M. (2013). Emotion Regulation Therapy for Generalized Anxiety Disorder. Cognitive and Behavioral Practice, 20(3), 282-300. http://doi.org/10.1016/j.cbpra.2013.02.001

[10] Fucito, L. M., Juliano, L. M., \& Toll, B. A. (2010). 
Cognitive Reappraisal and Expressive Suppression Emotion Regulation Strategies in Cigarette Smokers. Nicotine \& Tobacco Research, 12(11), 1156-1161. http://doi.org/10.1093/ntr/ntq146

[11] Gottschalk, M. G., \& Domschke, K. (2017). Genetics of generalized anxiety disorder and related traits. Dialogues in Clinical Neuroscience, 19(2), 159-168.

[12] Hasler, G., Fromm, S., Alvarez, R. P., Luckenbaugh, D. A., Drevets, W. C., \& Grillon, C. (2007). Cerebral Blood Flow in Immediate and Sustained Anxiety. The Journal of Neuroscience: The Official Journal of the Society for Neuroscience, 27(23), 6313-6319.

http://doi.org/10.1523/JNEUROSCI.5369-06.2007

[13] Hofmann, S. G., \& Smits, J. A. J. (2008). COGNITIVE-BEHAVIORAL THERAPY FOR ADULT ANXIETY DISORDERS: A META-ANALYSIS OF RANDOMIZED PLACEBO-CONTROLLED TRIALS. The Journal of Clinical Psychiatry, 69(4), 621-632.

[14] Lader, M. (2015). Generalized Anxiety Disorder. Encyclopedia of Psychopharmacology, 699-702. doi:10.1007/978-3-642-36172-2 317

[15] Levitis, D. A., Burger, O., \& Lackey, L. B. (2013). The human post-fertile lifespan in comparative evolutionary context. Evolutionary Anthropology: Issues, News, and Reviews, 22(2), 66-79. doi:10.1002/evan.21332

[16] Moyal, N., Henik, A., \& Anholt, G. E. (2013). Cognitive strategies to regulate emotions - current evidence and future directions. Frontiers in Psychology, 4, 1019. http://doi.org/10.3389/fpsyg.2013.01019

[17] Nowlan, J. S., Wuthrich, V. M., \& Rapee, R. M. (2015). The impact of positive reappraisal on positive (and negative) emotion among older adults. International Psychogeriatrics, 28(04), 681-693. doi:10.1017/s1041610215002057

[18] Ochi M, Fujiwara T, Mizuki R, Kawakami N. Association of socioeconomic status in childhood with major depression and generalized anxiety disorder: results from the World Mental Health Japan survey. BMC Public Health. 2014;14:1. doi: 10.1186/1471-2458-14-359.

[19] Pagliaccio, D., Luby, J. L., Bogdan, R., Agrawal, A., Gaffrey, M. S., Belden, A. C., Barch, D. M. (2015). Amygdala functional connectivity, HPA axis genetic variation, and life stress in children and relations to anxiety and emotion regulation. Journal of Abnormal Psychology, 124(4), 817-833. http://doi.org/10.1037/abn0000094
[20] Petrican, R., Rosenbaum, R. S., \& Grady, C. (2015). Expressive suppression and neural responsiveness to nonverbal affective cues. Neuropsychologia, 77, 321-330. http://doi.org/10.1016/j.neuropsychologia.2015.09.013

[21] Price, J. S. (2003). Evolutionary aspects of anxiety disorders. Dialogues in Clinical Neuroscience, 5(3), 223-236.

[22] R, K. (2016). Observing the Effects of Mindfulness-Based Meditation on Anxiety and Depression in Chronic Pain Patients. Abnormal and Behavioural Psychology, 02(01). doi:10.4172/2472-0496.1000111

[23] Reinhold, J. A., \& Rickels, K. (2015). Pharmacological treatment for generalized anxiety disorder in adults: An update. Expert Opinion on Pharmacotherapy, 16(11), 1669-1681. doi:10.1517/14656566.2015.1059424

[24] Renna, M. E., Quintero, J. M., Fresco, D. M., \& Mennin, D. S. (2017). Emotion Regulation Therapy: A Mechanism-Targeted Treatment for Disorders of Distress. Frontiers in Psychology, 8, 98. http://doi.org/10.3389/fpsyg.2017.00098

[25] Robinson, M. D., Watkins, E., \& Harmon-Jones, E. (2013). Handbook of cognition and emotion. New York: Guilford Press.

[26] Schlund, M. W., \& Cataldo, M. F. (2010). Amygdala involvement in human avoidance, escape and approach behavior. NeuroImage, 53(2), 769-776. http://doi.org/10.1016/j.neuroimage.2010.06.058

[27] Talaei-Khoei, M., Nemati-Rezvani, H., Fischerauer, S. F., Ring, D., Chen, N., \& Vranceanu, A. (2017). Emotion regulation strategies mediate the associations of positive and negative affect to upper extremity physical function. Comprehensive Psychiatry, 75, 85-93. doi:10.1016/j.comppsych.2017.03.005

[28] Waters AM., Bradley BP., Mogg K. Biased attention to threat in pediatric anxiety disorders (generalized anxiety disorder, social phobia, specific phobia, separation anxiety disorder) as a function of "distress" versus "fear" diagnostic categorization. Psychol Med. 2014;44(3):607-616. Doi: $10.1017 / \mathrm{S} 0033291713000779$

[29] Zhu, L.-J., Liu, M.-Y., Li, H., Liu, X., Chen, C., Han, Z., Zhou, Q.-G. (2014). The Different Roles of Glucocorticoids in the Hippocampus and Hypothalamus in Chronic Stress-Induced HPA Axis Hyperactivity. PLoS ONE, 9(5), e97689. http://doi.org/10.1371/journal.pone.0097689 